\title{
Beneficios de la ICC
}

\author{
Alfredo Milión \\ Director Ejecutivo de FUNDATEX y EXPORTEX
}

$\mathrm{E}$ n primer lugar quicro felicitar a los palrocinadores de este importinte evento por debalir el lemia de comercio y las reliaciones comerciales actuales y en segundo lugar agradecer y expresar que para mi es un verdadero honor estar con tan distinguido panel.

Quisiera hablar básicamente cle dos cosas. primero establecer que lugar ocupa Ia Iniciativa de la Cuenca del Caribe y la ampliación de sus benelicios dentro del contexio general de las relaciones comerciales yen segundo lugar entrar en los detalles particulares de algunos de los benelicios que en esta ocasión se obıuvieron con los EsIados Unidos.

Quisiera atreverme a decir que la Iniciativa de la Cuenca del Caribe o la ampliación de sus beneficios es. quizás, el cuarı paso dentro de lo que ha sido todo el rango de preferencia comerciales unilaterales a favor de paises en vías de desarrollo. Usıcdes recordarán que esto comienza al principio del establecimiento de los principios del GAP, donde a la par de todo ese esfuerzo nace un espacio para los paises en vías de desarrollo y comienza un proceso de otorgar un tratamiento especial a los países considerados menos desarrollados dentro del contexto mundial.

A sinace la ronda Kenedy y surge el sistema generalizado de preferencias, donde simplemente era un acceso comercial libre de aranceles de parte cle los países industrializados. Eso toma su propio cauce ya con la ampliación de la Iniciativa de la Cuenca del Caribe, es precisamente ese concepto general de los beneficios que ha hecho posible que naciones en viascle desarrollo, de los cuales los paises de Centroamérica y el Caribe no somos ninguna excepción, hallamos desarrollado los niveles de comercio y las exportaciones a los mercados de alıo consumo en particular Europa y los Estados Unidos.

\section{Productos libres de aranceles}

Aclualmente con la iniciativa de la Cuenca del Caribe. en lo que a nuestra región respecta, quizás hemos dado uno de los últimos pasos en la complementación de la agenda de acceso comercial, en donde más de cuatro mil productos quedaban excluidos, entre ellos: los textiles, algunos productos de cuero, atún y otros que no fueron incluidos en la primer iniciativa allá por $1974 y$ en la ampliacion de la misma a linales de los ochenta. Prácticamente. ahora llegamos al lener un acecso de merciados a los Estados Unidos. Salvo las condicionalidades: yuizis de los mais amplios yue puede cxistir en este momemo. Este lipo de alceseso hace posible no patgar impuestos en más de cuatro mil productos a lo largo de los aranceles actualmente en vigencia.

En segundo lugar los bencficios de la lniciativa de la Cuencal del Caribe. simple y sencillamente no llegall a alcamzar los niveles que nosotros hubieramos (querido) desde el punto de vista de acceso. En el contevio de la

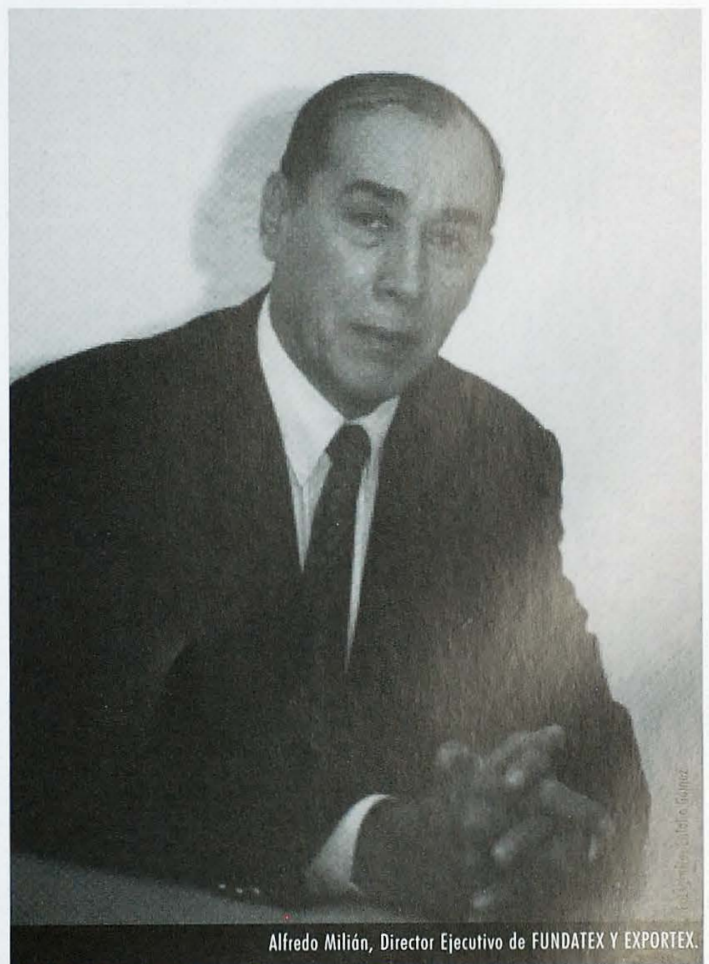


$\mathrm{OMC}$ el aı̃o dos mil cinco es el año final para poder llegar a una liberalización, a un verdadero estado de libre comercio. Eso nos coloca cn una situación muy difícil. el hecho de llegar a una elapa de libre comercio tiene que regocijarnos como tal, pero representa un reto mayor en el cual vamos a tener que competir dircctamente con paises como China que están llegando a unos niveles de cficiencia muy alıos.

Actualmente Centroamérica y el Caribe somos el sexto socio comercial más importante de los Estados Unidos, como el embajador René León había dicho, nosotros somos más importantes que el nivel de comercio que hay con los países de Sur America y con el resto de los paises del mundo, debido a una proximidad al mercado de los Estados Unidos y a la actitud politica que los Estados Unidos ha tomado con relación a estos paises.

Debo decir que no necesariamente somos un tema exclusivo de los Estados Unidos, de hecho, antc el reto de la perspectiva de la Unión Europea y de la formación de ese mega bloque comercial, sc gesió un Tratado de Libre Comercio de América del Norte, dentro del cuál México fue incluido. El trato comercial que México recibió directamente no excluyó las dos cláusulas sociales del NAFTA que son derechos laborales y medioambiente.

\section{Una zona privilegiada}

Debemos de entender una cosa y es que Estados Unidos seguirá tratando de introducir en la OMC esas dos cláusulas comerciales y dentro del ALCA igual, de manera que las condicionalidades que tenemos en la ICC son parte de esa filosofia puritana de la mentalidad de los Estados Unidos, dentro de lo que ellos consideran que son las relaciones comerciales y que van a seguir promoviendo hasta el año dos mil cinco.

Cualquiera podria pensar que China se va a escapar a eso, no creo. El tema de los derechos humanos es uno de los temas más difícil de discusión en el gobierno de los Estados Unidos y será con China una de las mayores dificultades para un tratamiento permanente, desde el punto de vista político. Cuando Estados Unidos de el siguiente paso para negociar otros tratados de libre comercio ya sea con paises en vias de desarrollo o con países desarrollados como la Unión Europea, estará sujeto a una aprobación del congreso de lo que se llama la via rápida. La via rápida es algo que tendrá el mismo tipo cle condicionamientos una vez entre al debate congresional.

Estamos viviendo una realidad, esta realidad es lo que hace posible que nuestros 24 países del Caribe $y$ Centroamerica tengamos esa perspectiva positiva, porque la regla del juego es quien tiene mayores preferencias de acceso antes del año dos mil cinco. Ahí se resume toda la venta ja comercial que cualquicr pais puede tener en estc momento con relación a un mercido como los Estados Unidos. El acceso al mercado que tanto Canddá siendo principal socio de los Estados Unidos y México obtuvieron en 1994. Ic da a estos paises una ventaja sobre cualquier negociación.

Pero quiero simplemente mencionar de que hay tres tipos de aranceles cle los Estados Unidos, el arancel nación más favorecida, el cual es un arancel general alto; el nivel cle las preferencias comerciales en el que entra NAFTA, CDI, Africa y que generalmente es libre; $y$ la columna dos del arancel, que es para los demás paises, especialmente aquelios con los que Estados Unidos no tiene relaciones economicas y comerciales, que es un arancel altisimo. Nosotros jugamos dentro de esos tres niveles de aranceles, el proposito principal es el llegar precisamente a la primera columna del arancel en donde no se paga impuesio.

Dentro de los productos textiles que fueron incorporados, a pesar cle que no logramos toda la verticalidad que hubiéramos querido lograr en término del tratamiento de telas por ejemplo, en los productos de tejido de punto y productos cle fibra sintética, estamos hablando que el arancel minimo es de 28:2\%, en el caso de las camisas de fibra sintética es del $32 \%$, en caso de blusas y camisas para mujeres de fibra sintética el $32 \%$ e igual cuando hablamos de camisetas. Estamos hablando de pagar una tercera parte de las Valorem en aranceles; ahora con la Iniciativa de la Cuenca del Caribc ese valor agregado, que es lo que generalmente tenemos, se liber aliza y llega a un cero por ciento eso es precisamente la ventaja que está causando en estos momentos toda la actitud positiva y la expectativa que nos da la perspectiva de las inversiones.

\section{Tela, hilo e hilaza norteamericana}

Ahora quiero hablar brevemente sobre que fue lo que conseguimos. Conseguimos el sector más preferencial de todos: el 807A especial, que se utiliza para ta maquila. Cuando se trata de tela hecha en Estados Unidos, con hilaza de Estados Unidos. cortada en Estados Uniclos y ensamblada en la región, ese valor agregado que generalmente en el caso de El Salvador representaba aproximadamente un $30 \%$, va a pagar ahora cero impuestos, esto es un rubro de los más importante en el rango general de la confección. $Y$ en segundo lugar a ese producto se le agregan todos los procesos que antes habian estado excluidos. como son los procesos de teñido, de tratamientos de (aces). estamos hablando de (termapres), del tratamiento de (encimas), en fin todo lo que es el tratamiento que antes descaliflcaba al $807 \mathrm{~A}$,pero que ahora está incluido. Esto va a representar quizás el rubro de mayor crecimiento en el corto plazo.

Luego tenemos un programa nuevo, se llama el $\$ 09$. Este va permitir que nosotros podamos traer tela hecha 
en Estados Unidess con hilaza de los Estados Unidos. pero que ya no se tenga que cortar en Estados Unidos, sino que se pucde cortar en espacios de Corte en El Salvador o en cualquier país que forme parte cle la iniciativa de la Cuenca del Caribe. La ICC genera una cosa muy interesante. que es similar a lo que era cl CDI, que el valor agregado que on el caso del CDI es cle $35 \%$ requisito para ganar la prelerencia cle milliples paises, cs decir que el valor agregado no necesariamente se tiene que incluir en un sólo país. sino que varios paises podrán incluirse en el proceso, de ahí que todos estos procesos se pueden hacer, una parte en El Sálvaclor. una parte cn Honduras. otra parte en Nicaragua y asi sucesivamente conseguir un mayor polencionamicnto de lo que son los procesos. Con el único requisito de que las prendas que se hagan con el programa $\mathrm{S} 09$ lienen que ser cosidas con hilos hechos en los Estados Unidos. Nosotros creemos que el $\$ 09$ va a ser quizás en el mediano plazo el sector de mayor crecimiento.

En tercer lugar estamos hablando de otros produclos, siempre de te jido de punto, que son de las exclusividades porque desgraciadamente dentro de las legislaciones se excluyo el tejido plano, por una razón muy simple. y es que la presencia política de los grupos de interés en esta legislación fue enorme, los textileros cle Estados Unidos insistieron en que el major valor está en el tejiclo plano. esas son las telas de mayor valor; consecuentemente fueron totalmente excluidas de la legislacion y se quedaron con el te jido de punto, que por cierio es más barato que el otro, paradarles una idea en términos generales en el comercio el tejido plano representa un $35 \%$ y el tejido de punto la diferencia. Pero en valor, el tejido plano represenla el $50 \%$ y el tejido de punto el otro $50 \%$. Ahí es donde algunos de los producios fueron considerados.

\section{Las cuotas tarifarias}

L.ucge vienen las cuotals larilarias yue si sce eslablecierencuando la legislación se planteci porprimeral ves, y era yue cl textil tenia yue recibir la preferencia en forma vertical couando un pais luviera la librat. Malivat. lal tela $y$ la prendia. pero lastimosimente esto no progresó y nos que-

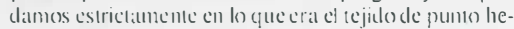
cho con hilaza de los Estados Unidos. esa fue la restricción yuc consegumios. Dentro de esis) se consiguicron dos cuotas (que son importantes. una es la de 250 millones de metros cenadrades eyuinalemes y el otro es de cuatro punto dos millones de dexenals de cilmuselas. esas son las dos cuolas tarif artias yue son con las yue lenemos yue trallary las dehemes compantir con lodos los palises de la incicialia de la Cuencal Jel Caribe.

Luego Icnemos otra disposicion que es lo que libera al calzado $y$ a otros productos, porque algunos de los producios que habian sido excluidos de la legislacion CDI, ahora se incorporaron en lo que es cl trataıniento de la desgravacion NAFTA. Es decir que cl calzado ahora entra $\mathrm{cn}$ el ramo cle las desgravaciones propias del NAF. TA. Con Mléxico liene otras disposiciones que son sumamente importantes y es que recoge del NAFTA la legislación de lo que son las telas en escasez de olerta. es decir que si existen telas que no están comercialmente disponibles en cantidades economicas. entonces se puede lograr una excepción. los paises podrán libremente importar telas. incluyendo telas chinas. para poder hacer confeccion y tendrian la misma preferencia. Esto es importante siempre y cuando haya aceptación de la escasez de las telas. Esta es una de las disposiciones v cláusulas que tienc Mixico

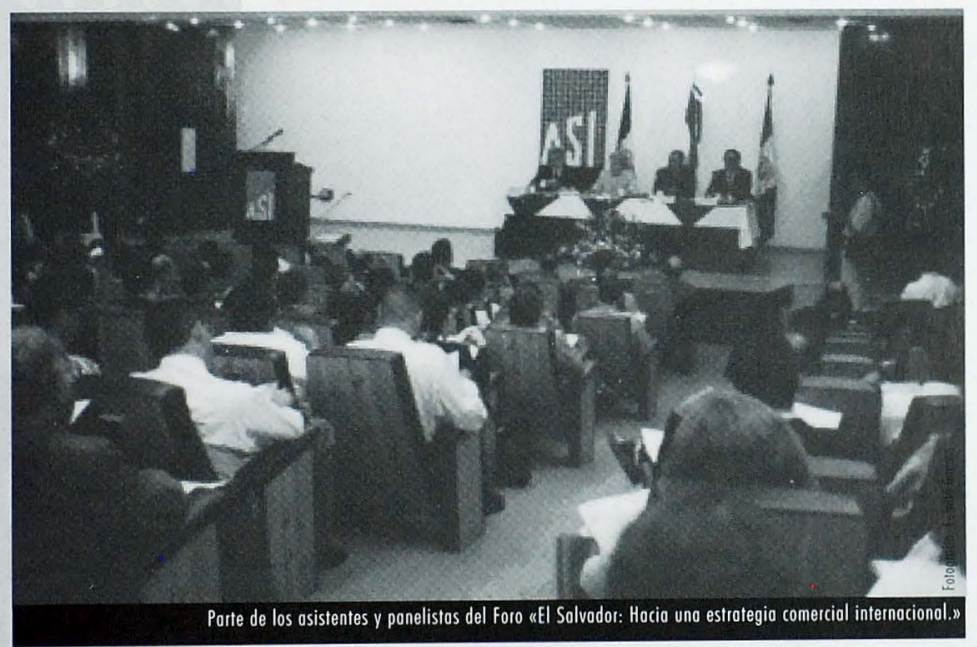




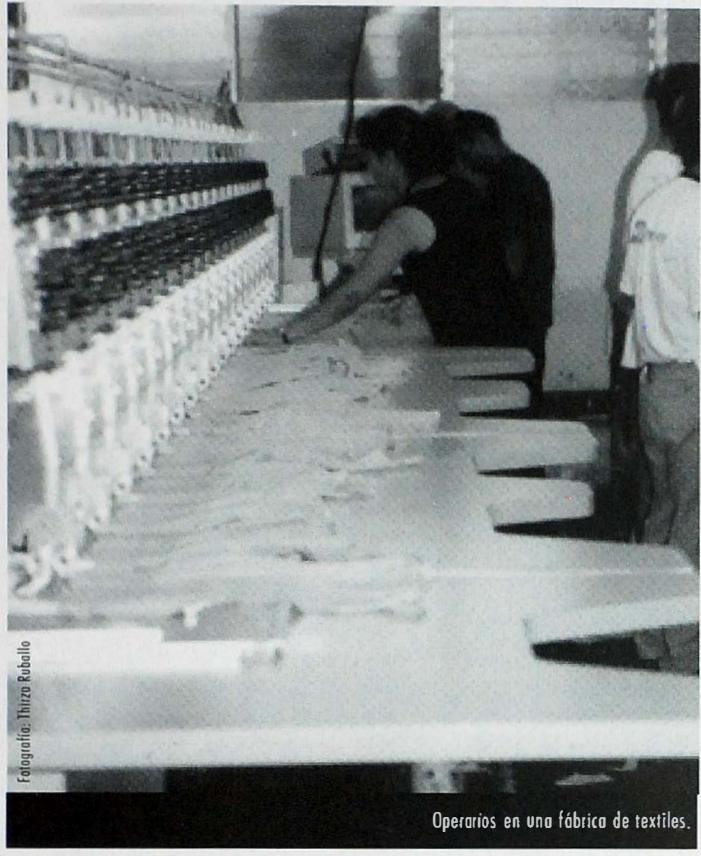

Productos artesanales

Paralos productos folklóricos y artesanales también hay una disposición de total liberalización. el único tema es que los productos folklóricos cstán todavía sujeıos a una mayor definición, eso es algo que Aduana de los Estados Unidos tiene que llegar a definir.

Tenemos luego las cláusulas de (minimes), porque se logró un nivel sin precedentes . cuando estamos halblando dentro del rango de la OMC, estamos hablando de que las cláusulas de (minimes) que son las de tolerancia para poder calificar un producto y poder incorporar ciertos niveles de productos extranjeros dentro del producto total. generalmente no pasin del $7 \%$. eso es lo que se negoció con México en el iratado de libre comercio. En este caso se consiguió un $25 \%$ al valorem, en lodo lo que son la combinación de cxcepciones que se incorporan y en términos de pesoparatelas y productos hechos de tela. nos quedamos en un $7 \%$ por peso. quiere decir que podemos meter encaljes. ziper , algunos clásticos. hilos botones. broches, dentro de los productos de la confección que van a gozar de la preferencia siempre y cuando los (Interlinen) que son los interiores de los cue llos y los puños y los demás no pasen del 25\%. Los Brassieres tienen Irato muy especial que ticne una regla de origen única en la cual requiere que $\mathrm{cl} 75 \%$ del valor de los brassieres tiene que ser de origen de los Estados Unidos. Básicamente hay ciertas disposiciones que limiIan una pequeña salvaguardia en los cuales se pierden las preferencias en caso de haber violación a los términos generales de la legislación.

Luego vienen las condicionalidades y la eligibilidad que es importantc. Básicamentc las condicionalidades que son once dentro cle la legislación no de jan de ser repetitıvas de algunas condicionalidades anteriores, por ejemplo el tema de los derechos laborales y el tema de derechos de propiedad intelectual están contenidos en la legislación CDI de 1984. inclusive el tema del ECGP. eso son temas que van a continuar $y^{\prime}$ que vamos a tener que vivir con ellos. Pero una de las cláusulas que son importantes y que incorporó el licenciado León. es que por primera vez en una legislación de los Estados Unidos queda explicito el mandato del congreso del presidente de los Estados Unidos de proceder, de manera inmediata. a abrir un foro de negociación con los paises de la Iniciatival Cuenca del Caribe.

Eso es importante, sobre todo dentro de la perspec liva politica por dos razones: porque gracias a Dios que logramos ia ampliación cle los beneficios de la 1CC a la terminación cle un gobierno demócrata, si ahora viene un gobierno demócrata nuevamente o viene un gobierno republicano, tiene la agenda completamente abierta para poder continuar con este segundo paso. Eso debe ser $\mathrm{cl}$ objelivo nuestro desde el punto de vista de política comercial. conseguir ese tratado de libre comercio con nuestro principal socio comercial, porque déjenme decirles esto. para todos los paises de la Iniciativa de la Cuenca del Caribe el principal socio es Estados Unidos. por grande. Entonces lograr un tratado de libre comercio con Estados Unidos, que casi ya lo tenemos va a ser muy diferente a las negociaciones con México. Número uno ya tenemos todo el acceso del mercado con casi la totalidad de los producios, son algunas excepciones. Ade más ya tenemos bastante adelantado los derechos de la propiedad intelectual, inversiones y una serie de conceptos que se manejan estrictamente en un tratado de libre comercio.

De manera que una negociación con EEUU dada las diferencias que tenemos y la complementariedad de las economias haria mucho más fácil un tratado de libre comercio. porque también lo estariamos manejando al nivel de varios paises. ese debería ser nuestro objetivo: lograrlo lo antes posible. porque repito la ventana para lograrlo se termina en el año dos mil cinco.

Y rinalmente el día de ayer apareció en el diario oficial de los Estados Unidos (Journal Register) las condiciones de lo que es el foro abierto para la elegibilidad ! si realmente los países vamos a poder cumplir con los requisitos establecidos por la leý, para poder ser beneticiarios de la legislación. Esıc es un proceso importantc que el gobierno le va a tener que dar lat matyor atención y también el sector empresarial para ver en que meclida nosotros cumplimos con esto y hacerlo lo antes posible Quiero terminar aqui rápidamente esta prescntación esperar alguna pregunta de parte del público. gracias. 